\title{
The Bounds for Eigenvalues of Normalized Laplacian Matrices and Signless Laplacian Matrices
}

\author{
Serife Büyükköse', Sehri Gülçiçek Eski² \\ ${ }^{1}$ Department of Mathematics, Faculty of Science, Gazi University, Ankara, Turkey \\ ${ }^{2}$ Institue of Science, Ahi Evran University, Kırşehir, Turkey \\ Email: serifebuyukkose@gmail.com, gulcicekeski@gmail.com
}

Received 8 September 2014; revised 6 October 2014; accepted 5 November 2014

Copyright (C) 2014 by authors and Scientific Research Publishing Inc.

This work is licensed under the Creative Commons Attribution International License (CC BY).

http://creativecommons.org/licenses/by/4.0/

c. (i) Open Access

\begin{abstract}
In this paper, we found the bounds of the extreme eigenvalues of normalized Laplacian matrices and signless Laplacian matrices by using their traces. In addition, we found the bounds for $k$-th eigenvalues of normalized Laplacian matrix and signless Laplacian matrix.
\end{abstract}

Keywords

Normalized Laplacian Matrix, Signless Laplacian Matrix, Bounds of Eigenvalue

\section{Introduction}

Let $G(V, E)$ be a simple graph with the vertex set $V=\left\{v_{1}, v_{2}, \cdots, v_{n}\right\}$ and edge set of $E$. For $v_{i} \in V$, the degree of $v_{i}$, the set of neighbours of $v_{i}$ are denoted by $d_{i}$ and $N_{i}$, respectively. If $v_{i}$ and $v_{j}$ are adjacent, we denote $v_{i} \sim v_{j}$ of short use $i \sim j$.

The adjacency matrix, Laplacian matrix and diagonal matrix of vertex degree of a $G$ graph are denoted by $A(G), L(G), D(G)$, respectively. Clearly

$$
L(G)=D(G)-A(G)
$$

The normalized Laplacian matrix of $G$ is defined as $\mathscr{L}(G)=D^{-1 / 2}(G) L(G) D^{-1 / 2}(G)$ i.e., $\mathscr{L}(G)=\left[\ell_{i j}\right]_{n \times n}$ where

$$
\ell_{i j}=\left\{\begin{array}{ccc}
1 & ; & \text { if } i=j \\
\frac{-1}{\sqrt{d_{i} d_{j}}} & ; & \text { if } i \sim j \\
0 & ; & \text { otherwise. }
\end{array}\right.
$$

How to cite this paper: Büyükköse, S. and Eski, S.G. (2014) The Bounds for Eigenvalues of Normalized Laplacian Matrices and Signless Laplacian Matrices. Advances in Linear Algebra \& Matrix Theory, 4, 201-204. 
The signless Laplacian matrix of $G$ is defined as $Q(G)=D(G)+A(G)$ i.e., $Q(G)=\left[q_{i j}\right]_{n \times n}$ where

$$
q_{i j}=\left\{\begin{array}{ccc}
d_{i} & ; \quad \text { if } i=j \\
1 & ; \quad \text { if } i \sim j \\
0 & ; & \text { otherwise. }
\end{array}\right.
$$

Since $\mathscr{L}(G)$ normalized Laplacian matrix and $Q(G)$ signless Laplacian matrix are real symetric matrices, their eigenvalues are real. We denote the eigenvalues of $\mathscr{L}(G)$ and $Q(G)$ by

$$
\lambda_{1}(\mathscr{L}(G)) \geq \cdots \geq \lambda_{n}(\mathscr{L}(G))
$$

and

$$
\lambda_{1}(Q(G)) \geq \cdots \geq \lambda_{n}(Q(G))
$$

respectively.

Now we give some bounds for normalized Laplacian matrix and signless Laplacian matrix.

1. Oliveira and de Lima's bound [1]: For a simple connected graph $G$ with $n$ vertices and $m$ edges, $\Delta=d_{1} \geq d_{2} \geq \cdots \geq d_{n}=\delta$

$$
\lambda_{1}(Q(G)) \leq \max _{i}\left\{\frac{d_{i}+\sqrt{d_{i}^{2}+8 d_{i} m_{i}}}{2}\right\}
$$

where $m_{i}=\frac{1}{d_{i}} \sum_{i \sim j} d_{j}$.

\section{Another Oliveira and de Lima's bound [1]:}

$$
\lambda_{1}(Q(G)) \leq \max _{i}\left\{d_{i}+\sqrt{d_{i} m_{i}}\right\}
$$

where $m_{i}=\frac{1}{d_{i}} \sum_{i \sim j} d_{j}$.

\section{Li, Liu et al. bound's [2] [3]:}

$$
\lambda_{1}(Q(G)) \leq \frac{\Delta+\delta-1 \sqrt{(\Delta+\delta-1)^{2}+8(2 m-(n-1) \delta)}}{2}
$$

4. Rojo and Soto's bound [4]: If $\lambda_{1}$ is the largest eigenvalue of $\mathscr{L}$ then

$$
\left|\lambda_{1}(\mathscr{L}(G))\right| \leq 2-\min _{i<j}\left(\frac{\left|N_{i} \cap N_{j}\right|}{\max \left\{d_{i}, d_{j}\right\}}\right)
$$

where the minimum is taken over all pairs $(i, j), \quad(1 \leq i<j \leq n)$.

In this paper, we found extreme eigenvalues of normalized Laplacian matrix and signless Laplacian matrix of a $G$ graph with using theirs traces.

To obtain bounds for eigenvalues of $\mathscr{L}(G)$ and $Q(G)$ we need the followings lemmas and theorems.

Lemma 1. Let $W$ and $\lambda=\left(\lambda_{j}\right)$ be nonzero column vectors, $e=(1,1, \cdots, 1)^{\mathrm{T}}, C=I_{n}-\frac{e e^{\mathrm{T}}}{n} m=\frac{\lambda^{\mathrm{T}} e}{n}$, $s^{2}=\frac{\lambda^{\mathrm{T}} C \lambda}{n}$ and $I_{n}$ is an identity matrix. Let $\lambda_{1} \geq \lambda_{2} \geq \cdots \geq \lambda_{n}$. Then,

$$
\begin{aligned}
& -s \sqrt{n W^{\mathrm{T}} C W} \leq W^{\mathrm{T}} \lambda-m W^{\mathrm{T}} e=W^{\mathrm{T}} C \lambda \leq s \sqrt{n W^{\mathrm{T}} C W} \\
& \sum_{j}\left(\lambda_{j}-\lambda_{n}\right)^{2}=n\left[s^{2}+\left(m-\lambda_{n}\right)^{2}\right]
\end{aligned}
$$




$$
\begin{aligned}
& \sum_{j}\left(\lambda_{1}-\lambda_{j}\right)^{2}=n\left[s^{2}+\left(\lambda_{1}-m\right)^{2}\right] \\
& \lambda_{n} \leq m-\frac{s}{\sqrt{n-1}} \leq m+\frac{s}{\sqrt{n-1}} \leq \lambda_{1}
\end{aligned}
$$

Theorem 1 [5]. Let $A$ be a $n \times n$ complex matrix. Conjugate transpose of $A$ denoted by $A^{*}$. Let $B=A A^{*}$ whose eigenvalues are $\lambda_{1}(B) \geq \lambda_{2}(B) \geq \cdots \geq \lambda_{n}(B)$. Then

and

$$
m-s \sqrt{n-1} \leq \lambda_{n}^{2}(B) \leq m-\frac{s}{\sqrt{n-1}}
$$

$$
m+\frac{s}{\sqrt{n-1}} \leq \lambda_{1}^{2}(B) \leq m+s \sqrt{n-1}
$$

where $m=\frac{\operatorname{tr} B}{n}$ and $s^{2}=\frac{\operatorname{tr} B^{2}}{n}-m$.

\section{Main Results for Normalized Laplacian Matrix}

Theorem 2. Let $G$ be a simple graph and $\mathscr{L}(G)$ be a normalized Laplacian matrix of $G$. If the eigenvalues of $\mathscr{L}(G)$ are $\lambda_{1}(\mathscr{L}(G)) \geq \lambda_{2}(\mathscr{L}(G)) \geq \cdots \geq \lambda_{n}(\mathscr{L}(G))$, then

$$
\begin{aligned}
& \lambda_{n} \mathscr{L}(G) \leq \sqrt{\left(1+\frac{2}{n_{i \sim j, i<j}} \sum_{i} \frac{1}{d_{i} d_{j}}\right)+\sqrt{\frac{\operatorname{tr}[L(G)]^{4}-n m^{2}}{n(n-1)}}} \\
& \lambda_{1} \mathscr{L}(G) \geq \sqrt{\left(1+\frac{2}{n_{i \sim j, i<j}} \frac{1}{d_{i} d_{j}}\right)+\sqrt{\frac{\operatorname{tr}[L(G)]^{4}-n m^{2}}{n(n-1)}}} \\
& \lambda_{1} \mathscr{L}(G) \leq \sqrt{1+\frac{2}{n_{i \sim j, i<j}} \sum_{i} \frac{1}{d_{i} d_{j}}+\sqrt{\left(\frac{\operatorname{tr}[L(G)]^{4}}{n}-m^{2}\right)(n-1)}}
\end{aligned}
$$

Proof. Clearly

$$
\operatorname{tr}[\mathscr{L}(G)]^{2}=n+2 \sum_{i \sim j, i<j} \frac{1}{d_{i} d_{j}}
$$

and

$$
\operatorname{tr}[\mathscr{L}(G)]^{4}=\sum_{i=1}^{n}\left(1+\sum_{i \sim j} \frac{1}{d_{i} d_{j}}\right)^{2}+2 \sum_{i<j}\left(\sum_{k \in N_{i} \cap N_{j}} \frac{1}{d_{k} \sqrt{d_{i} d_{j}}}-\sum_{i \sim j} \frac{2}{\sqrt{d_{i} d_{j}}}\right)^{2}
$$

Since $\mathscr{L}(G)$ real symmetric matrix, we found the result from Theorem 1.

Example 1. Let $G=(V, E)$ with $V=\{1,2,3,4,5,6\}$ and $E=\{(1,2),(1,5),(2,3),(2,4),(2,6),(3,4),(3,5),(4,5),(5,6)\}$

\begin{tabular}{cccc}
\hline$\lambda(\mathcal{C}(G))$ & $(4)$ & (6) (lower bound) & (7) (upper bound) \\
\hline 1.86 & 2 & 1.34 & 1.93 \\
\hline
\end{tabular}

\section{Main Results for Signless Laplacian Matrix}

Theorem 3. Let $G$ be a simple graph and $Q(G)$ be a signless Laplacian matrix of $G$. If the eigenvalues of 
$Q(G)$ are $\lambda_{1}(Q(G)) \geq \lambda_{2}(Q(G)) \geq \cdots \geq \lambda_{n}(Q(G))$, then

$$
\begin{aligned}
& \lambda_{n}(Q(G)) \leq \sqrt{\left(1+\frac{2}{n_{i \sim j, i<j}} \frac{1}{d_{i} d_{j}}\right)+\sqrt{\frac{\operatorname{tr}[Q(G)]^{4}-n m^{2}}{n(n-1)}}} \\
& \lambda_{1}(Q(G)) \geq \sqrt{\left(1+\frac{2}{n_{i \sim j, i<j}} \frac{1}{d_{i} d_{j}}\right)+\sqrt{\frac{\operatorname{tr}[Q(G)]^{4}-n m^{2}}{n(n-1)}}} \\
& \lambda_{1}(Q(G)) \leq \sqrt{1+\frac{2}{n_{i \sim j, i<j}} \sum_{i} \frac{1}{d_{i} d_{j}}+\sqrt{\left.\frac{\operatorname{tr}[Q(G)]^{4}}{n}-m^{2}\right)(n-1)}}
\end{aligned}
$$

Proof. Clearly

$$
\operatorname{tr}[Q(G)]^{2}=n+2 \sum_{i \sim j, i<j} \frac{1}{d_{i} d_{j}}
$$

and

$$
\operatorname{tr}[Q(G)]^{4}=\sum_{i=1}^{n}\left(1+\sum_{i \sim j} \frac{1}{d_{i} d_{j}}\right)^{2}+2 \sum_{i<j}\left(\sum_{k \in N_{i} \cap N_{j}} \frac{1}{d_{k} \sqrt{d_{i} d_{j}}}-\sum_{i \sim j} \frac{2}{\sqrt{d_{i} d_{j}}}\right)^{2}
$$

Since $Q(G)$ was real symmetric matrix, we found the result from Theorem 1 .

Example 2. Let $G=(V, E)$ with $V=\{1,2,3,4,5,6,7\}$ and $E=\{(1,2),(1,3),(1,4),(1,5),(1,6),(1,7),(2,3),(3,5),(4,5),(4,6)\}$

\begin{tabular}{cccccc}
\hline$\lambda(Q(G))$ & $(1)$ & $(2)$ & $(3)$ & (9) (lower bound) & (10) (upper bound) \\
\hline 7.67 & 9.08 & 9.74 & 9.34 & 4.58 & 7.76 \\
\hline
\end{tabular}

\section{References}

[1] Oliveira, C.S., De Lima, L.S., De Abreu, N.M.M. and Hansen, P. (2010) Bound on the Index of the Signless Laplacian of a Graph. Discrete Applied Mathematics, 158, 355-360. http://dx.doi.org/10.1016/j.dam.2009.06.023

[2] Li, J. and Pan, Y. (2004) Upper Bounds for the Laplacian Graph Eigenvalues. Acta Mathematica Sinica, English Series, 20, 803-806. http://dx.doi.org/10.1007/s10114-004-0332-4

[3] Liu, H., Lu, M. and Tian, F. (2004) On the Laplacian Spectral Radius of a Graph. Linear Algebra and Its Applications, 376, 135-141. http://dx.doi.org/10.1016/j.laa.2003.06.007

[4] Rojo, O. and Soto, R.L. (2013) A New Upper Bound on the Largest Normalized Laplacian Eigenvals. Operators and Matrices, 7, 323-332. http://dx.doi.org/10.7153/oam-07-19

[5] Wolkowich, H. and Styan, G.P.H. (1980) Bounds for Eigenvalues Using Traces of Matrice. Linear Algebra and Its Applications, 29, 471-506. http://dx.doi.org/10.1016/0024-3795(80)90258-X 
Scientific Research Publishing (SCIRP) is one of the largest Open Access journal publishers. It is currently publishing more than 200 open access, online, peer-reviewed journals covering a wide range of academic disciplines. SCIRP serves the worldwide academic communities and contributes to the progress and application of science with its publication.

Other selected journals from SCIRP are listed as below. Submit your manuscript to us via either submit@scirp.org or Online Submission Portal.
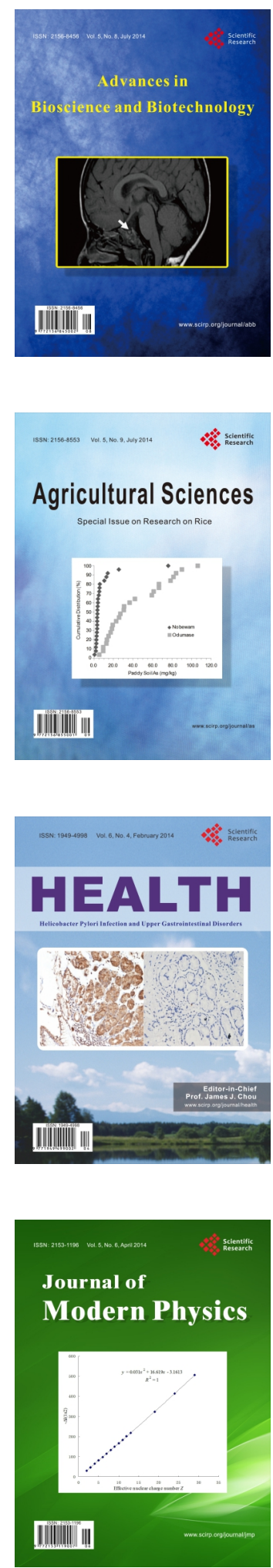
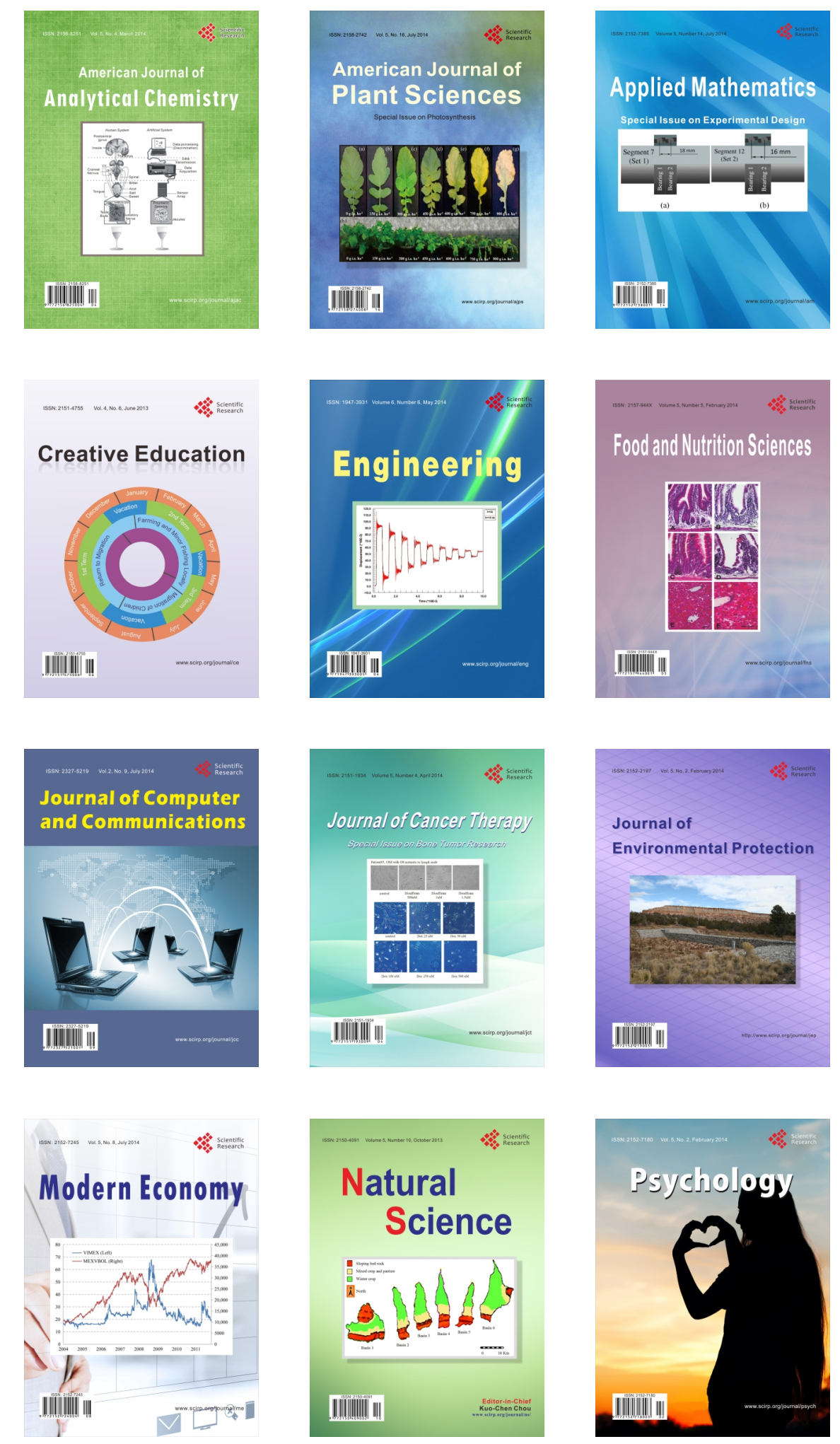